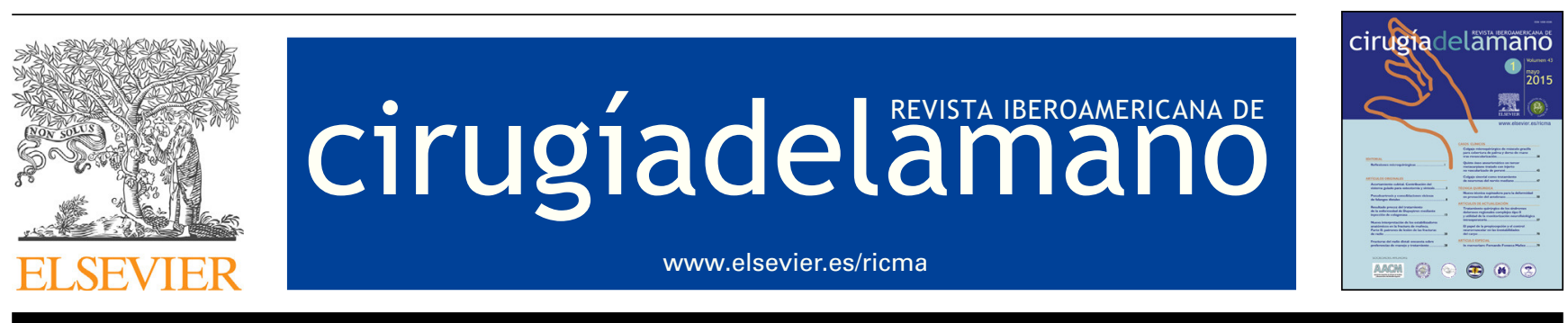

ARTÍCULO ORIGINAL

\title{
Valores normativos de la fuerza de puño en la población española en edad laboral. Influencia de las variables antropométricas de la mano y el antebrazo
}

\author{
J.A. Oteo ${ }^{a, *}$, P. Benavente ${ }^{a}$ y M. Garzón ${ }^{b}$ \\ a Servicio de Cirugía Ortopédica y Traumatología, Hospital Universitario de Fuenlabrada, Madrid, España \\ b Departamento de Anatomía, Histología y Neurociencia, Universidad Autónoma de Madrid, Madrid, España
}

Recibido el 12 de mayo de 2015; aceptado el 29 de septiembre de 2015

Disponible en Internet el 3 de octubre de 2015

\section{PALABRAS CLAVE \\ Fuerza de puño; \\ Apertura \\ dinamómetro; \\ Parámetros \\ antropométricos}

\begin{abstract}
Resumen
Objetivos: Determinar si en los individuos adultos sanos existe una apertura óptima del dinamómetro para conseguir la máxima fuerza de puño y si esta se relaciona con los valores de los parámetros antropométricos del antebrazo y la mano. Saber si a través de las medidas antropométricas del antebrazo y la mano es posible predecir la fuerza de puño que puede desarrollar un persona.

Métodos: 99 hombres y 103 mujeres (20-60 años). Cada mano se testó 10 veces, utilizando 5 aperturas del dinamómetro TKK. Las medidas antropométricas tomadas fueron ancho de la mano abierta, longitud del segundo, el tercer y el cuarto dedos, anchura de la palma, longitud de la palma, longitud de la mano, longitud del antebrazo, perímetro del antebrazo y grosor de la eminencia tenar.

Resultados: Con solo 2 aperturas (5,5-6 cm en varones, $5-5,5 \mathrm{~cm}$ en mujeres) se consiguió la máxima fuerza de puño en más del $60 \%$ de los individuos. El tamaño de la mano y el antebrazo influyó en la posición de apertura óptima en las mujeres, no en los varones. Los parámetros antropométricos que mejor definieron la fuerza fueron diferentes según el sexo y el lado, pero en ningún caso se logró que el valor de $\mathrm{R}^{2}$ superase el 0,50 .

Conclusiones: La máxima fuerza de puño se consigue utilizando las posiciones medias del dinamómetro, siendo en mujeres la apertura menor que en varones. Empleando exclusivamente los parámetros antropométricos de la mano y el antebrazo no se consigue un valor predictivo útil, como el obtenido por otros autores con otras medidas antropométricas.

(C) 2015 SECMA. Publicado por Elsevier España, S.L.U. Este es un artículo Open Access bajo la licencia CC BY-NC-ND (http: // creativecommons.org/licenses/by-nc-nd/4.0/).
\end{abstract}

\footnotetext{
* Autor para correspondencia.

Correo electrónico: oteom@yahoo.com (J.A. Oteo).
} 


\section{KEYWORDS}

Handgrip strength;

Dynamometer span; Anthropometric measures
Securities regulatory force fist in Spanish working age population. Anthropometric influence of variables of the hand and forearm

\begin{abstract}
Objetives: To determine in adult healthy subjects if some particular grip spans of a dynamometer have the same competence to reach the maximum handgrip strength and, if so, whether those optimal grip spans are related to anthropometric measures of hand and forearm. To examine the association between handgrip strength and anthropometric measures of hand and forearm, to recognize parameters defining better a subject's handgrip.

Methods: 99 men and 103 women (age 20-60) were evaluated. Each hand was randomly tested on 10 trials using 5 grip spans with TKK dynamometer. The anthropometric measures taken were hand span, second, third and fourth fingers length, palm width, palm length, hand length, forearm length, forearm circumference and adductor pollicis longs thickness.

Results: With only two grip spans $(5,5-6 \mathrm{~cm}$ in men, $5-5,5 \mathrm{~cm}$ in women) attain more than $60 \%$ of the maximum handgrip strength. The optimal grip span and anthropometric measures of hand and forearm correlated only in women, not in men. Anthropometric parameters that best define handgrip strength were different in men and women, and between sides, but the value of $\mathrm{r} 2$ did not exceed 0,50 of the predictive ability in any case.

Conclusions: To obtain maximum grip strength is assumed to use middle positions of the dynamometer, but women need smaller openings than men. Using hand and forearm parameters, we do not get as good results as others using anthropometric parameters.

( 2015 SECMA. Published by Elsevier España, S.L.U. This is an open access article under the CC BY-NC-ND license (http://creativecommons.org/licenses/by-nc-nd/4.0/).
\end{abstract}

\section{Introducción}

La dinamometría es un modo objetivo de medir la fuerza muscular realizada por un individuo, consiguiendo expresarla como una variable cuantitativa, lo que facilita su valoración. Además es un método sensible, fácil y rápido de realizar, con un coste moderado, y reproducible (siempre que los instrumentos de medida estén bien calibrados y se estandarice la toma), lo que hace que actualmente sea el sistema más adecuado para evaluar de forma objetiva la fuerza muscular ${ }^{1,2}$.

La fuerza de puño es un indicador muy útil de la situación general de salud de un individuo, dado que cifras bajas se han asociado al síndrome metabólico ${ }^{3}$, la malnutrición ${ }^{4,5}$, la osteoporosis $^{6}$ y la insuficiencia renal ${ }^{7}$, entre otras patologías, e incluso es un predictor consistente de la mortalidad en adultos y ancianos ${ }^{8}$. En cirugía de la mano es una herramienta básica para el control de diferentes patologías ${ }^{7,8}$.

Sin embargo, conocer cuáles son los valores de referencia es complicado. Las bases de datos agrupadas por edad y sexo son muy dependientes del tipo de población y del país. Otra opción que se emplea es predecir la fuerza de puño utilizando los parámetros antropométricos del individuo a estudio, especialmente han sido usados el peso, la altura o el índice de masa corporal, pero en ocasiones, tales como pacientes encamados, esto es complejo de conocer.

Teniendo en cuenta lo comentado anteriormente, el presente estudio tiene los siguientes objetivos:

- Establecer los valores de fuerza de puño en la población general española en edad laboral sobre la base del mayor tamaño muestral publicado hasta la fecha.

- Saber qué posiciones del dinamómetro son las óptimas para realizar la medición del mismo y si existe relación entre estas posiciones y el tamaño de la mano y el antebrazo a estudio.

- Saber si empleando exclusivamente las medidas antropométricas de la mano y el antebrazo se puede explicar la variabilidad de la fuerza de puño que puede desarrollar una persona.

\section{Material y método}

El diseño clínico fue el de un estudio descriptivo de corte transverso. Se realizó la toma de datos durante 3 meses en un grupo de 202 voluntarios sanos, entre la población que acudió como acompañante a las consultas del Hospital Universitario de Fuenlabrada (99 varones, 103 mujeres) residentes en varias ciudades del sur de la Comunidad Autónoma de Madrid (España); no se excluyó ninguna raza, todos los participantes estaban en edad laboral (20-60 años) y se confirmó tras realizar una entrevista que no presentaban patología sistémica grave ni disfunción neuromuscular u ortopédica que pudieran afectar la fuerza de puño. Todas las entrevistas y las mediciones fueron realizadas por la misma persona, quien proporcionaba a los participantes información detallada acerca del propósito de la investigación y de las implicaciones de la misma, además de estimularles durante la prueba para que hicieran el máximo esfuerzo posible.

Se tomaron datos de edad, sexo y dominancia (según la mano que utilizara el sujeto para escribir), así como varias medidas antropométricas de la mano y el antebrazo (hechas con una cinta de medición inextensible y flexible): ancho de la mano abierta (en cm, distancia entre la punta del pulgar y la del quinto dedo con los dedos extendidos y la mano lo más abierta posible), longitud del segundo, el tercer y el cuarto dedos de la mano (en centímetros, distancia entre el pliegue 
palmar metacarpo-falángico y la punta del dedo en cuestión), ancho de la palma (en centímetros, distancia a nivel de la cabeza de los metacarpianos), longitud de la palma (en centímetros, distancia entre el pliegue de la muñeca hasta el pliegue de la articulación metacarpo-falángica), longitud de la mano (en centímetros, distancia entre el pliegue de la muñeca y la punta del tercer dedo), longitud del antebrazo (en centímetros, distancia entre olécranon y apófisis estiloides cubital con el codo en flexión de $90^{\circ}$ y el antebrazo en posición neutra) perímetro del antebrazo (en centímetros, perímetro en la zona de la unión entre el tercio proximal y medio con el codo en flexión de $90^{\circ}$ y el antebrazo en posición neutra) y grosor de la eminencia tenar (en milímetros, realizado con el plicómetro Holtain LTD [Crymych, Reino Unido], se midió a nivel de vértice del ángulo imaginario formado por la prolongación del borde cubital del pulgar y el borde radial del segundo dedo).

Para efectuar la medición de la fuerza de puño (en kilogramos), se empleó un dinamómetro digital de mano (T.K.K. 540 Grip-D, Takey, Tokio, Japón) (fig. 1) considerado entre los dinamómetros manuales el que tiene una mayor validez y reproducibilidad ${ }^{9}$. Durante las mediciones los sujetos permanecían en bipedestación, con el hombro en abducción leve $\left(10^{\circ}\right.$ aproximadamente), el codo en extensión completa y el antebrazo y la mano en posición neutra (fig. 2). Cada persona efectuó (alternativamente con ambas manos) el test 2 veces con cada una de las diferentes posiciones posibles de apertura del dinamómetro, de forma aleatoria y dejando un minuto de descanso entre las medidas. Las aperturas utilizadas fueron $4,5,5,0,5,5,6,0,6,5$ y 7,0 cm. Si la medida del ancho de la mano abierta era $<20 \mathrm{~cm}$, la apertura

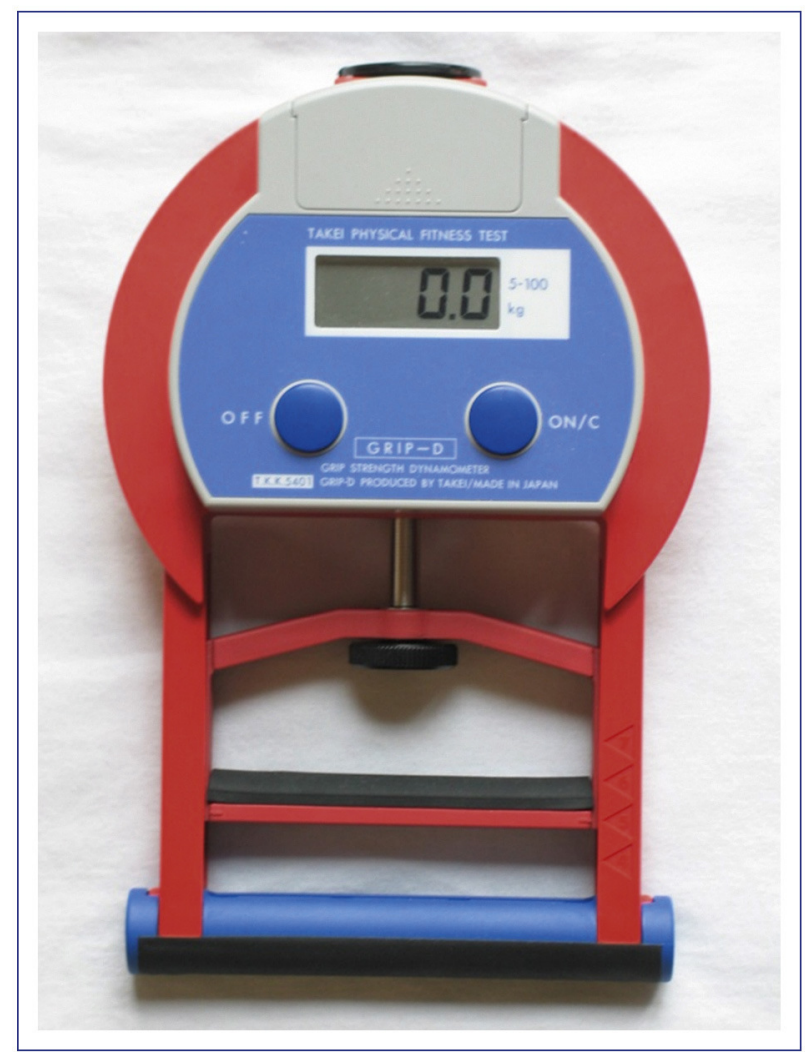

Figura 1 Dinamómetro T.K.K. 540 Grip-D, Takey, Tokio, Japón. mayor $(7,0)$ se rechazaba; si, por el contrario, esta medida era $>20 \mathrm{~cm}$, la que se rechazaba era la menor $(4,5)$.

Para la determinación de la fuerza de puño máxima se realizó una gráfica donde se relacionaba la fuerza con la apertura del dinamómetro para cada individuo y cada mano, encontrando 3 tipos de relación: cuadrático (donde la fuerza y la apertura tenían una relación cuadrática y parabólica, ajustada a un polinomio de segundo grado) en 103 individuos en el lado derecho y 105 en el izquierdo; lineal (donde se obtenía más fuerza a mayor apertura) en 34 individuos en el lado derecho y 27 en el izquierdo, e irregular (no había relación entre la apertura del dinamómetro y la fuerza desarrollada) en 65 individuos en el lado derecho y en 70 en el izquierdo, tomándose en este caso el valor medio.

Dado que los valores de fuerza no tenían una distribución normal en la totalidad de la población a estudio, se decidió emplear pruebas no paramétricas para el análisis estadístico, el cual se realizó con el programa informático SPSS 17.0 (SPSS Inc. Chicago, IL, EE. UU.). Para valorar muestras relacionadas (la fuerza entre ambos lados y el valor máximo y mínimo en un mismo individuo), se utilizó el test de Wilcoxon, mientras que para 2 muestras independientes (la fuerza y la apertura del dinamómetro entre varones y mujeres) se usó el test $U$ de Mann-Whitney. El test de Kruskal-Wallis sirvió para contrastar el valor de fuerza y la edad, así como la apertura del dinamómetro y el tamaño de la mano y el antebrazo. El estadístico rho de Spearman se usó para conocer si

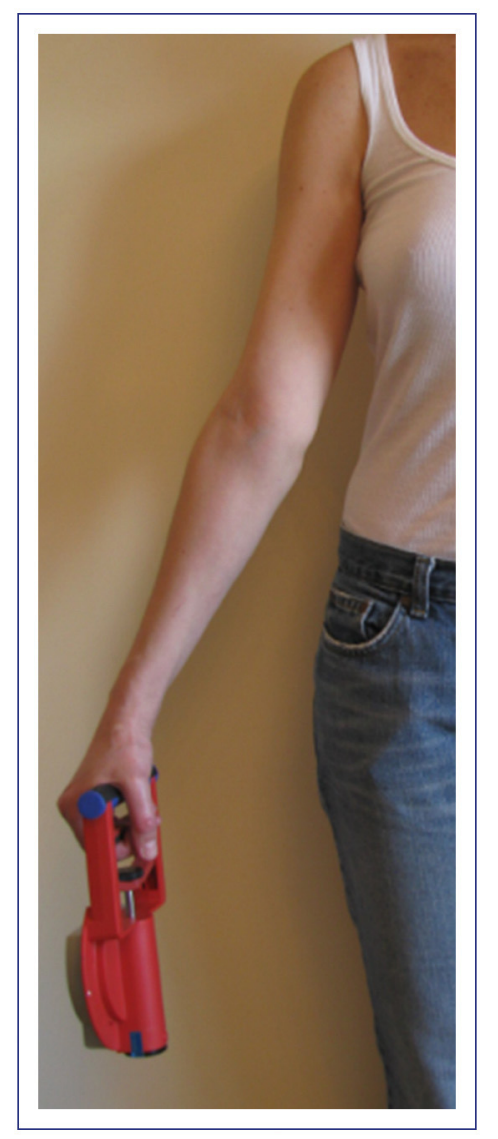

Figura 2 Posición del sujeto durante la realización de las mediciones. 
Tabla 1 Fuerza máxima, sexo y lado

\begin{tabular}{llll} 
& Varones & Mujeres & \multicolumn{1}{c}{} \\
\hline Lado derecho & $47,3 \pm 7(30,5-66,7)^{\mathrm{a}}$ & $29,2 \pm 4,7(19,9-42,1)^{\mathrm{a}}$ & $<0,001^{\mathrm{b}}$ \\
Lado izquierdo & $43,4 \pm 6,8(24,2-59)^{\mathrm{a}}$ & $27,6 \pm 5(18,6-39,7)^{\mathrm{a}}$ & $<0,001^{\mathrm{b}}$ \\
$\mathrm{p}$ & $<0,001^{\mathrm{c}}$ & $0,001^{\mathrm{c}}$ & \\
\hline
\end{tabular}

a Datos dados con desviación típica y rango entre paréntesis.

b Test U de Mann-Whitney. La distribución de la variable fuerza de puño es diferente a través de las categorías varón/mujer.

c Test de Wilcoxon. La distribución de la variable fuerza de puño es diferente a través de las categorías lado derecho/izquierdo.

la diferencia de fuerza máxima y la mínima se relacionaba con el nivel de fuerza del individuo.

Para conocer si era posible construir un modelo que explicara la variabilidad de la fuerza de puño (variable dependiente), según los diferentes parámetros antropométricos de la mano y el antebrazo (variables independientes), se realizó una regresión lineal múltiple (paramétrica), buscando aquel que maximizara el coeficiente de determinación $\mathrm{R}^{2}$, con el menor número de variables, eliminando aquellas que solo producen mínimos incrementos en $\mathrm{R}^{2}$.

\section{Resultados}

Respecto a los valores de fuerza y sexo (tabla 1), se observaron diferencias estadísticamente significativas entre ambos lados y ambos sexos.

Solo 9 participante eran zurdos (6 varones y 3 mujeres). Cuando la mano derecha era la dominante, la fuerza de este lado en relación con el no dominante era mayor un 7,5\% en varones y un 6,3\% mujeres; cuando era el izquierdo el dominante, esta diferencia se reducía, no existiendo en las mujeres y siendo de un 3,3\% en varones.

En las tablas $2 a$ y $2 b$ se muestran los valores de fuerza de puño según sexo y edad (divididos por grupos homogéneos en número de individuos). Los valores de fuerza fueron superiores en el grupo de edad entre 31-35 años en todos los casos, salvo en varones en el lado izquierdo, en ellos la mayor fuerza se conseguía en el grupo entre 36-40 años. Estas diferencias por edad no eran estadísticamente significativas, salvo en el lado izquierdo de las mujeres.

La diferencia de fuerza en cada individuo entre el valor de fuerza máximo y mínimo obtenido con las mediciones realizadas con las diferentes aperturas del dinamómetro, fue estadísticamente significativa $(p<0,001)$. En el varón dicha diferencia era de $9,4 \mathrm{~kg}$ en lado derecho y de $9,2 \mathrm{~kg}$ en lado izquierdo, mientras que en la mujer era de 6,4 en ambos lados. El valor máximo y mínimo presentaba una correlación estadísticamente significativa en ambas manos $(p<0,001)$, esto es, cuanta mayor fuerza realizaba un individuo, más diferencia entre el valor máximo y el mínimo existía, aunque este valor fue siempre bajo (en varones 0,383 en el lado derecho y 0,395 en lado izquierdo, mientras que en mujeres era 0,370 en lado derecho y 0,326 en lado izquierdo).

Más del $75 \%$ de los participantes en el estudio consiguieron la máxima fuerza de puño con 3 aperturas $5,5,5$ y $6 \mathrm{~cm}$ ( $80,2 \%$ en lado derecho y $78,2 \%$ en lado izquierdo). Había una diferencia estadísticamente significativa entre ambos sexos en relación con la apertura del dinamómetro $(p<0,001)$. En los varones, la máxima fuerza de puño se obtuvo con las aperturas de 5,5 y $6 \mathrm{~cm}$ en un $60,6 \%$ de los casos en el lado derecho y en un 59,6\% en el lado izquierdo; por el contrario, con estas aperturas solo se consiguió la menor fuerza de puño en un $9,1 \%$ en el lado derecho y en un $20,2 \%$ en el lado izquierdo (tabla 3). En las mujeres se consiguió la máxima fuerza de puño con las aperturas del dinamómetro de 5 y $5,5 \mathrm{~cm}$ en un $68 \%$ de los casos en el lado derecho y en un $57,3 \%$ en el lado izquierdo; con estas mismas aperturas solo se consiguió la menor fuerza de puño en un $15,5 \%$ de los casos en el lado derecho y en un $18,4 \%$ en el lado izquierdo.

La apertura del dinamómetro donde se lograba la máxima fuerza de puño se distribuía de forma similar en los varones, no importando las diferentes medidas de los parámetros de la mano y el antebrazo, mientras que en las mujeres no fue así; en ellas la distribución de la apertura óptima del dinamómetro no era igual según algunos parámetros, que eran más numerosos y con una significación estadística mayor en el lado izquierdo (ancho de la mano abierta $[p<0,001]$, longitud de segundo $[p<0,001]$, tercer $[p<0,001]$ y cuarto $[p<0,001]$ dedos, longitud de la mano $[p=0,006]$ y longitud de antebrazo $[p=0,006]$ ) que en el derecho (longitud de la palma $[p=0,036]$, longitud de la mano $[p=0,03]$ y longitud de antebrazo [p = 0,033]).

Cuando se aplicó la regresión lineal múltiple (paramétrica), el modelo que obtenía un valor mayor de $\mathrm{R}^{2}$ era diferente según el sexo y el lado; en los varones, fueron la longitud del tercer dedo y el perímetro del antebrazo, a los que se añadía el ancho de la mano abierta en el lado derecho, mientras que en las mujeres fueron la longitud del tercer dedo y la longitud del antebrazo, a los que se añadían, en el lado derecho, el perímetro del antebrazo y, en el lado izquierdo, el grosor del abductor pollicis longus. La capacidad para explicar la variabilidad de la fuerza de puño de estos modelos resultó muy baja; el valor del coeficiente de correlación obtenido $\left(\mathrm{R}^{2}\right)$ no superó en ninguno de los casos 0,50 (varones lado derecho 0,41 y lado izquierdo 0,25 ; mujeres lado derecho 0,43 y lado izquierdo 0,37 ).

\section{Discusión}

Presentamos los valores normativos de fuerza de puño en la población general española adulta en edad laboral, con un tamaño muestral de 202 individuos, lo que supera en número otros estudios publicados anteriormente ${ }^{10}$. Se vio que las posiciones medias del dinamómetro servían para conseguir la máxima fuerza de puño y que esta posición no dependía del tamaño de la mano y el antebrazo en el varón pero si en la mujer. Asimismo se observó que los parámetros de la mano y el antebrazo, por sí solos, no eran útiles para explicar la variabilidad de la fuerza de puño. 
Tabla 2a Fuerza máxima y edad en varones

\begin{tabular}{|c|c|c|c|c|c|c|c|c|c|c|c|}
\hline \multicolumn{2}{|c|}{ Varones } & \multicolumn{5}{|c|}{ Lado derecho } & \multicolumn{5}{|c|}{ Lado izquierdo } \\
\hline Edad, años & N. ${ }^{\circ}$ casos & Media \pm DT & Mediana & Rango & Percentil 25 & Percentil 75 & Media \pm DT & Mediana & Rango & Percentil 25 & Percentil 75 \\
\hline $20-30$ & 21 & $47,78 \pm 6,33 \pm$ & 47,4 & 23,3 & 42,5 & 53,35 & $44,28 \pm 5,9$ & 42,9 & 20,3 & 38,7 & 48,7 \\
\hline $31-35$ & 14 & $51,31 \pm 4,13$ & 50,5 & 14,8 & 48,4 & 53,82 & $46,85 \pm 3,97$ & 46,25 & 12,1 & 43,42 & 51 \\
\hline $36-40$ & 21 & $50,91 \pm 7,39$ & 50,5 & 23,7 & 44,4 & 56,45 & $47,08 \pm 7,07$ & 47,8 & 27,1 & 42,1 & 51 \\
\hline $41-50$ & 25 & $49,32 \pm 8,47$ & 49,7 & 36,6 & 44,65 & 54 & $46 \pm 7,58$ & 47,1 & 32,5 & 42,1 & 50,85 \\
\hline $51-60$ & 18 & $46,33 \pm 4,56$ & 46,55 & 15,8 & 43 & 50 & $42,53 \pm 4,47$ & 42,15 & 16,1 & 38,77 & 46,37 \\
\hline $\mathrm{p}$ & & \multicolumn{5}{|c|}{$0,104^{a}$} & \multicolumn{5}{|c|}{$0,079^{a}$} \\
\hline
\end{tabular}

a Test de Kruskal-Wallis. La distribución de la fuerza a través de las diferentes categorías de edad es similar en ambos lados en varones.

Tabla 2b Fuerza máxima y edad en mujeres

\begin{tabular}{|c|c|c|c|c|c|c|c|c|c|c|c|}
\hline \multicolumn{2}{|c|}{ Mujeres } & \multicolumn{5}{|c|}{ Lado derecho } & \multicolumn{5}{|c|}{ Lado izquierdo } \\
\hline Edad, años & N. ${ }^{\circ}$ casos & Media \pm DT & Mediana & Rango & Percentil 25 & Percentil 75 & Media \pm DT & Mediana & Rango & Percentil 25 & Percentil 75 \\
\hline $20-30$ & 19 & $29,87 \pm 3,43$ & 29,7 & 12,2 & 28 & 32,5 & $27,68 \pm 4,13$ & 28 & 17,9 & 24,8 & 30,3 \\
\hline $31-35$ & 19 & $31,94 \pm 5,09$ & 31 & 21,3 & 29,1 & 35,3 & $30,47 \pm 4,5$ & 30,1 & 16,2 & 27,3 & 34 \\
\hline $36-40$ & 22 & $30,29 \pm 3,68$ & 30,45 & 14,8 & 27,77 & 32,8 & $28,79 \pm 3,44$ & 28,25 & 12,5 & 26,4 & 31,82 \\
\hline $41-50$ & 25 & $30,04 \pm 5,33$ & 29,1 & 19,2 & 25,7 & 36,15 & $27,85 \pm 4,84$ & 28,6 & 17,6 & 24 & 31,25 \\
\hline $51-60$ & 18 & $27,96 \pm 4,38$ & 26,9 & 15,4 & 25,17 & 29,87 & $25,92 \pm 3,37$ & 25,7 & 14,2 & 23,37 & 27,17 \\
\hline $\mathrm{p}$ & & & $0,099^{a}$ & & & & & $0,015^{a}$ & & & \\
\hline
\end{tabular}

a Test de Kruskal-Wallis. La distribución de la fuerza a través de las diferentes categorías de edad es similar en el lado derecho de las mujeres, pero no en el izquierdo. 
Tabla 3 Apertura del dinamómetro donde se consigue la fuerza máxima

\begin{tabular}{lccccc} 
& \multicolumn{2}{c}{ Varones } & & \multicolumn{2}{c}{ Mujeres } \\
\cline { 2 - 3 } \cline { 5 - 6 } Apertura $(\mathrm{cm})$ & Lado dcho & Lado izdo & & Lado dcho & Lado izdo \\
\hline 4,5 & 0 & 1 & & 14 & 13 \\
5 & 21 & 20 & & 30 & 28 \\
5,5 & 33 & 28 & & 40 & 31 \\
6 & 27 & 31 & & 11 & 20 \\
6,5 & 14 & 14 & & 8 & 10 \\
7 & 4 & 5 & & 0 & 1 \\
\hline
\end{tabular}

Cuando se analizó la fuerza en ambos sexos, nuestro estudio halló una diferencia estadísticamente significativa favorable a los varones, siendo la fuerza de la mujer ligeramente superior al $60 \%$ del valor de la fuerza en el varón, lo que sigue los hallazgos encontrados por otros autores ${ }^{11-13}$.

Aunque la mano dominante (por lo general, la derecha) suele tener más fuerza que la no dominante (tradicionalmente se ha considerado que es de un $10 \%$ superior $^{14}$ ), la diferencia varía bastante según el artículo que se valore ${ }^{12,15}$. En nuestro caso, la máxima diferencia la encontramos en varones diestros $(7,3 \%)$ y menor en personas zurdas, al igual que han visto otros autores ${ }^{16}$.

Respecto a los valores obtenidos según la edad, se sabe que hay una asociación curvilínea de la fuerza de puño con la edad, con un aumento a partir de la adolescencia, sobre todo en varones ${ }^{17}$, alcanzando su máximo en la treintena ${ }^{12,16}$ para luego disminuir, siendo este descenso significativo a partir de los 60 años $^{18}$. En nuestra revisión, los hallazgos corroboraron estos datos, dado que la máxima fuerza se consiguió en la tercera década de la vida, aunque no se apreciaron diferencias estadísticamente significativas en nuestra población que iba de 20 a 60 años, salvo en la mano izquierda de las mujeres, quizás debido a la menor fuerza que desarrollaban.

La American Society of Hand Therapy (ASHT) recomienda la segunda posición del dinamómetro Jamar $(4,8 \mathrm{~cm})$ para realizar los estudios, dado que tradicionalmente se considera esta la posición para conseguir más fuerza ${ }^{14}$, mientras que Härkönen et al. consideran una mejor opción usar la tercera posición del Jamar $(6 \mathrm{~cm})^{19}$. Nosotros encontramos que las posiciones centrales son las ideales para conseguir la máxima fuerza de puño y en las que hay una menor posibilidad de conseguir un valor mínimo, apreciando que las mujeres consiguen mejores resultado utilizando un rango de apertura menor que el varón, coincidiendo con lo ya publicado por Peolsson et al. ${ }^{11}$, que recomiendan el uso de la segunda posición del Jamar para las mujeres y la tercera para los varones.

Se sabe que existe una posición óptima del dinamómetro para conseguir un valor máximo de fuerza y que depende del tamaño de la mano en mujeres adultas (también en niños y adolescentes de ambos sexos), pero no en varones adultos, posiblemente por tener estos una mayor masa muscular ${ }^{10,20,21}$. En nuestro análisis, se confirmó que solo en las mujeres la distribución de la posición óptima de la apertura de puño era diferente según el tamaño de la mano y el antebrazo.

Se asume que cuando una persona realiza un esfuerzo máximo la variación entre el valor máximo y mínimo debe ser menor de $20 \%$ y, generalmente, menor del $10 \%{ }^{14}$. En nuestro caso, esa diferencia no superó nunca el $10 \%$, siendo mayor cuanto mayor era la fuerza máxima del individuo.

Hanten et al. ${ }^{22}$, empleando parámetros genéricos como género, altura, peso, edad y dominancia, consiguen explicar entre el 61 - 62\% de la variabilidad en la fuerza de puño en varones y mujeres entre 20 y 64 años. Vaz et al. ${ }^{23}$ utilizan como predictores el sexo, la edad y la altura, además del perímetro del antebrazo, y con ello consiguen conocer la fuerza de puño en el 63,3\% de los individuos mayores de 18 años. Günther et al. ${ }^{12}$, usando valores genéricos como la edad, el sexo, la altura y el índice de masa corporal, consiguen predecir la fuerza de puño en adultos hasta un $76,2 \%$ en el lado derecho y un $75,2 \%$ en el lado izquierdo. Frente a estos datos, en nuestro estudio, usando sólo parámetros de antebrazo y mano, no se consigue explicar ni el $50 \%$ de la variabilidad de la fuerza de puño. Vimos que los parámetros que mejor definían la fuerza eran diferentes dependiendo del sexo y el lado que se trataba, siendo la longitud del tercer dedo el único parámetro que se repetía en todos los casos. En esto último se coincide con Visnapuu y Jürimae ${ }^{24}$, quienes encuentran que la longitud de los dedos, en especial el tercero y el cuarto, es el factor local que más influye en la fuerza de la mano, por ser el más implicado en el agarre del dinamómetro, la continuación natural del antebrazo y, por tanto, la línea primaria de transmisión de fuerza desde el radio.

Para terminar, nos gustaría destacar dos limitaciones importantes del estudio; en primer lugar, el tamaño muestral que, aunque supera a los publicados previamente, posiblemente no llegue a reflejar la variabilidad de la población española adulta en edad laboral y, en segundo lugar, el no haber tomado medidas antropométricas generales, como peso o talla, además de las del antebrazo y la mano, para poder conocer cómo podrían mejorar la definición de los valores de fuerza de puño.

\section{Conclusión}

Se aportan valores normativos de la fuerza de puño en la población adulta española, teniendo el mayor tamaño muestral publicado hasta la fecha. Se ha visto que para conseguir la fuerza máxima de puño de un individuo debemos utilizar una apertura de dinamómetro entre 5 y $5,5 \mathrm{~cm}$ en las mujeres y entre 5,5 y 6 en los varones; esta posición óptima depende del tamaño de la mano en las mujeres, especialmente en su lado izquierdo, pero no en los varones, debido probablemente a la mayor fuerza de estos. Además, se ha valorado que el uso exclusivo de los parámetros antropométricos de la mano y el antebrazo no sirve para explicar la variabilidad de la fuerza de puño.

\section{Conflicto de intereses}

Los autores declaran no tener ningún conflicto de intereses.

\section{Bibliografía}

1. Huesa Jimenez F, García Diez J, Vargas Montes J. Dinamometría isocinética. Rehabilitación. 2005;39(6):288-96. 
2. Hogrel JY, Ollivier G, Desnuelle C. Testing musculaire manuel et quantifié dans les maladies neuromusculaires. Comment assurer la qualitédes measures de force dans les protocols cliniques? Rev Neurol A (París). 2006;162(4):427-36.

3. Aihie Sayer A, Syddall HE, Dennison EM, Martin HJ, Phillips DIW, Cooper C, et al. The relationship between grip strength and features of the metabolic syndrome: Findings from Hertfordshire Cohort Study. QJM. 2007;100(11):707-13.

4. Chilima DM, Ismail SJ. Nutrition and handgrip strength of older adults in rural Malawi. Public Health Nutr. 2000;4(1):11-7.

5. Chevalier S, Saoud F, Gray-Donald K, Morais JA. The physical functional capacity of frail elderly persons undergoing ambulatory rehabilitation is related to their nutritional status. J Nutr Health Aging. 2008;12(10):721-6.

6. Dixon WG, Lunt M, Pye SR, Reeve J, Felsenberg D, Silman AJ, et al. Low grip strength is associated with bone mineral density and vertebral fracture in women. Rheumatology (Oxford). 2005;44(5):642-6.

7. Guo Q, Bárány P, Qureshi AR, Snaedal S, Heimbürger O, Stenvinkel $P$, et al. $\mathrm{N}$-terminal pro-brain natriuretic peptide independently predicts protein energy wasting and is associated with all-cause mortality in prevalent HD patients. Am J Nephrol. 2008;29(6):516-23.

8. Al Snih S, Markides KS, Ray L, Ostir GV, Goodwin JS. Handgrip strength and mortality in older Mexican American. J Am Geriatr Soc. 2002;50(7):1250-6.

9. España-Romero V, Ortega FB, Vicente-Rodriguez G, Artero EG, Rey P, Ruiz JR. Elbow position affects handgrip strength in adolescents: Validity and reability of Jamar, DynEx and TKK dynamometers. J Strength Cond Res. 2010;24(1): 272-7.

10. Ruiz-Ruiz J, Mesa JL, Gutierrez A, Castillo MJ. Hand size influences optimal grip span in women but not in men. J Hand Surg Am. 2002;27(5):897-901.

11. Peolsson A, Hedlund R, Oberg B. Intra- and inter-tester reliability and reference values for values for hand strength. J Rehabil Med. 2001;33(1):36-41.

12. Günther CM, Bürger A, Rickert M, Crispin A, Schulz CU. Grip strength in healthy Caucasian adults: Reference values. J Hand Surg Am. 2008;33(4):558-65.
13. Kuh D, Bassey J, Butterworth S, Hardy R, Wadsworth ME, the Musculoskeletal Study Team. Grip strength, postural control, and functional leg power in a representative cohort of British men and women: associations with physical activity, health status, and socioeconomic conditions. J Gerontol A Biol Sci Med Sci. 2005;60(2):224-31.

14. Innes E. Handgrip strength testing: A review of the literature. Austral OccupTher J. 1999;46(3):120-40.

15. Clerke A, Clerke J. A literature review of the effect of handedness on isometric grip strength differences of the left and right hands. Am J OccupTher. 2001;55:206-11.

16. Massy-Westropp N, Rakin W, Ahern M, Krishnan J, Hearn TC. Measuring grip strength in normal adults: reference ranges and comparison of electronic a hydraulic instruments. J Hand Surg Am. 2004;29(3):514-9.

17. Sartorio A, Lafortuna CL, Pogliaghi S, Trecate L. The impact of gender, body dimension and body composition on hand-grip strength in healthy chidden. J Endocrinol Invest. 2002;25(5):431-5.

18. Bassey EJ, Harreis UJ. Normal values for handgrip strength in 920 men and women aged over 65 years, and longitudinal changes over 4 years in 620 survivors. Clin Sci. 1993;84(3):331-7.

19. Härkönen R, Piirtomaa M, Araranta H. Grip strength ans hand position of the dynamometer in 204 Finnish adults. J Hand Surg Br. 1993;35(3):551-69.

20. Ruiz JR, España-Romero V, Ortega FB, Sjöström M, Castillo MJ, Gutierrez A. Hand span influences optimal grip span in male and female teenagers. J Hand Surg Am. 2006;31(8):1367-72.

21. España-Romero V, Artero EG, Santaliestra-Pasias AM, Gutierrez A, Castillo MJ, Ruiz JR. Hand span influences optimal grip span in boys and girls aged 6 to 12 years. J Hand Surg Am. 2008;33(3):378-84.

22. Hanten WP, Chen WY, Austin AA, Brooks RE, Carter HC, Law CA, et al. Maximum grip strength in normal subjects from 20 to 64 years of age. J Hand Ther. 1999;12(3):193-200.

23. Vaz M, Hunsberger S, Diffey B. Prediction equations for handgrip strength in healthy Indian male and female subjects encompassing a wide age range. Ann Hum Biol. 2002;29(2):131-41.

24. Visnapuu M, Jürimae T. Handgrip strength and hand dimensions in young handball and basketball players. J Strength Cond. 